\title{
ON ABSTRACT FUBINI THEOREMS FOR FINITELY ADDITIVE INTEGRATION
}

\author{
E. DE AMO AND M. DÍAZ CARRILLO
}

(Communicated by Andrew M. Bruckner)

\begin{abstract}
A Fubini theorem for positive linear functionals on the vector lattice of the real-valued functions is given. This result properly contains that of the Riemann- $\mu$-abstract integral.
\end{abstract}

\section{INTRODUCTION}

In [3] one starts with a functional $I: B \rightarrow \mathbb{R}$, defined on the vector lattice $B$ of real-valued functions on a set $X$ and assumed to be positive linear. One defines the extended function class $R_{1}(B, I)$ and extends $I$ to $R_{1}(B, I)$ via one or the other of three classical methods (certain limits of elementary functions, equality of the upper and lower integrals, closure of $B$ with respect to an $R_{1}$ type seminorm), and one gets the convergence theorems using a suitable "local convergence in measure". Riemann- $\mu$, abstract Riemann-Loomis and Bourbaki integrals are subsumed.

In [5] Elsner has given a Fubini type theorem for the abstract Riemann- $\mu$ integral. In this note, the integral extension of Lebesgue power introduced in [2] and [3] is used to develop a Fubini type theorem in quite general settings.

Let $I_{1}$ and $I_{2}$ be positive linear functionals on vector lattices over $X_{1}$ and $X_{2}$, respectively. A methodological simplification is obtained by constructing the iterated integrals, via a suitable extension of the linear functionals. Conditions are determined for an integrable function $f: X_{1} \times X_{2} \rightarrow \overline{\mathbb{R}}$, without assuming continuity, so that the iterated integrals exist and are equal. So, our results are a reasonable substitute for Fubini's theorem for finitely additive integration (or for corresponding to the analogues to the Daniell extension process, but without continuity assumptions on the elementary integral $I$ ).

\section{Product Systems}

On the extended real line $\overline{\mathbb{R}}=\mathbb{R} \cup\{-\infty, \infty\}$ we adopt the usual conventions $0( \pm \infty):=0$ and $\infty+(-\infty):=0$. We denote $a \vee b:=\max (a, b), a \wedge b:=$ $\min (a, b), a, b \in \overline{\mathbb{R}}$.

Terminology and notation used are similar to that of [2], [3] and [10].

Received by the editors November 10, 1993 and, in revised form, January 24, 1994.

1991 Mathematics Subject Classification. Primary 28A35, 28 C05.

Key words and phrases. Fubini theorem, finitely additive measure, abstract Riemann integral. 
(1) Throughout this note we shall assume that for $j=1,2, X_{j}$ is an arbitrary set, $B_{j} \subset R^{X_{j}}$ a vector lattice (with respect to pointwise operations) and $I_{j}: B_{j} \rightarrow \mathbb{R}$ a linear functional which is positive, i.e., $I_{j}(f) \geq 0$ for all $f \geq 0$ in $B_{j}$.

Let $X_{3}:=X_{1} \times X_{2}$ and $B_{3} \subset R^{X_{3}}$ a vector lattice. For $f \in \overline{\mathbb{R}}^{X_{3}}$ and for $x \in X_{1}$ we define the function $f_{x}$ on $X_{2}$ by $f_{x}(y)=f(x, y)$ for each $y \in X_{2}$. Let $f$ be a function on $X_{3}$ such that $f_{x} \in B_{2}$ for each $x \in X_{1}$. Then setting $\left(I_{2} f\right)(x):=I_{2}\left(f_{x}\right)$ for each $x \in X_{1}$, we have defined the function $I_{2} f$ on $X_{1}$.

(2) A system $\left(X_{3}, B_{3}\right)$ is called a product system with respect to $\left(X_{1}, B_{1}\right)$ and $\left(X_{2}, B_{2}\right)$, whenever for each $f \in B_{3}$ the following conditions are satisfied:

(i) $f_{x} \in B_{2}$ for each $x \in X_{1}$.

(ii) $I_{2} f \in B_{1}$.

In all that follows $\left(X_{3}, B_{3}\right)$ will be a product system. We define a positive linear functional on $B_{3}$ by the rule $I_{3}(f):=I_{1}\left(I_{2} f\right)$ for each $f \in B_{3}$.

\section{The abstract Fubini theorem}

2.1. Proper Riemann integration. (3) For $f \in \overline{\mathbb{R}}^{X_{j}}, j=1,2,3$, we define Riemann upper and lower integrals by

$I_{j}^{-}(f):=\inf \left\{I_{j}(h) ; f \leq h \in B_{j}\right\}$, with $\inf \varnothing:=\infty$ and $I_{j}^{+}(f):=-I_{j}^{-}(-f)$.

$I_{j}^{-}$is positively homogeneous and subadditive on $\overline{\mathbb{R}}^{X_{j}}$.

For $f \in \overline{\mathbb{R}}^{X_{3}}$ we define the function $I_{2}^{-} f: X_{1} \rightarrow \overline{\mathbb{R}}$ by $\left(I_{2}^{-} f\right)(x):=I_{2}^{-}\left(f_{x}\right)$ for each $x \in X_{1}$. Similarly, $\left(I_{2}^{+} f\right)(x):=-I_{2}^{-}\left(-f_{x}\right)$.

Lemma 1. If $f \in \overline{\mathbb{R}}^{X_{3}}$, then $I_{1}^{-}\left(I_{2}^{-} f\right) \leq I_{3}^{-}(f)$ and $I_{3}^{+}(f) \leq I_{1}^{+}\left(I_{2}^{+} f\right)$.

Proof. By (3) and (ii) of (2), one has $I_{3}^{-}(f):=\inf \left\{I_{1}\left(I_{2} h\right) ; f \leq h \in B_{3}\right\} \geq$ $\inf \left\{I_{1}\left(I_{2} h\right) ; I_{2}^{-} f \leq I_{2}^{-} h=I_{2} h, h \in B_{3}\right\} \geq \inf \left\{I_{1}(g) ; I_{2}^{-} f \leq g \in B_{1}\right\}=$ : $I_{1}^{-}\left(I_{2}^{-} f\right)$.

The rest of the proof is similar.

(4) The set $R_{\text {prop }}\left(B_{j}, I_{j}\right)$ of proper Riemann integrable functions is defined as the set of those functions $f \in \mathbb{R}^{X_{j}}$ such that any one of the following conditions, which are equivalent, is satisfied.

(i) Given any $\varepsilon \in \mathbb{R}^{+}$, there exist $h, g \in B_{j}$ such that $I_{j}(h-g)<\varepsilon$, with $g \leq f \leq h$.

(ii) $I_{j}^{+}(f)=I_{j}^{-}(f) \in \mathbb{R}$.

We have that $R_{\text {prop }}\left(B_{j}, I_{j}\right)$ is the closure of $B_{j}$ with respect to the integral seminorm $I_{j}^{-}(|\cdot|)$. If $f \in R_{\text {prop }}\left(B_{j}, I_{j}\right), I_{j}(f):=I_{j}^{+}(f)=I_{j}^{-}(f)$ (see, for example, [1], [2]).

$A \subset X_{j}$ is called an $I_{j}^{-}$-null set iff $I_{j}^{-}\left(\chi_{A}\right)=0$.

Theorem 1. If $f \in R_{\text {prop }}\left(B_{3}, I_{3}\right)$, then:

(i) $I_{2}^{-} f, I_{2}^{+} f \in R_{\text {prop }}\left(B_{1}, I_{1}\right)$.

(ii) There exist $A_{k} \subset X_{1}, k \in \mathbb{N}, I_{1}^{-}$-null sets, such that $f_{x} \in R_{\text {prop }}\left(B_{2}, I_{2}\right)$ for all $x \in X_{1}-\bigcup_{1}^{\infty} A_{k}$.

(iii) There exists $g \in R_{\text {prop }}\left(B_{1}, I_{1}\right)$ defined by $I_{2}^{-}\left(f_{x}\right)$ if $f_{x} \in R_{\text {prop }}\left(B_{2}, I_{2}\right)$, and such that $I_{3}(f)=I_{1}(g)$. 
Proof. (i) For $f \in R_{\text {prop }}\left(B_{3}, I_{3}\right)$, by (4), (3) and Lemma 1, we have

$$
I_{3}(f):=I_{3}^{-}(f) \geq I_{1}^{-}\left(I_{2}^{-} f\right) \geq\left\{\begin{array}{l}
I_{1}^{-}\left(I_{2}^{+} f\right) \\
I_{1}^{+}\left(I_{2}^{-} f\right)
\end{array}\right\} \geq I_{1}^{+}\left(I_{2}^{+} f\right) \geq I_{3}^{+}(f):=I_{3}(f) .
$$

Then, $I_{3}(f)=I_{1}^{-}\left(I_{2}^{-} f\right)=I_{1}^{+}\left(I_{2}^{-} f\right) \in \mathbb{R}$, and by (4) $I_{2}^{-} f \in R_{\text {prop }}\left(B_{1}, I_{1}\right)$. Similarly, $I_{2}^{+} f \in R_{\text {prop }}\left(B_{1}, I_{1}\right)$.

(ii) For $x \in X_{1}$, set $h(x):=I_{2}^{-}\left(f_{x}\right)-I_{2}^{+}\left(f_{x}\right)$. One has $0 \leq h \in R_{\text {prop }}\left(B_{1}, I_{1}\right)$ and $I_{1}(h)=0$.

Now, let $A_{k}:=\left\{x \in X_{1} ; h(x) \geq \frac{1}{k}\right\}, k \in \mathbb{N}$. Since $I_{1}^{-}\left(\chi_{A_{k}}\right) \leq k I_{1}(h)=0$, $A_{k}$ are $I_{1}^{-}$-null sets, and by (4), $f_{x} \in R_{\text {prop }}\left(B_{2}, I_{2}\right)$ for all $x \in X_{1}-\bigcup_{1}^{\infty} A_{k}$.

(iii) Finally, if $g \in \overline{\mathbb{R}}^{X_{1}}$ such that $I_{2}^{-}\left(f_{x}\right) \leq g(x) \leq I_{2}^{+}\left(f_{x}\right)$ for all $x \in$ $X_{1}-\bigcup_{1}^{\infty} A_{k}$, then, by (4), we obtain $g \in R_{\text {prop }}\left(B_{1}, I_{1}\right)$ and $I_{1}(g)=I_{1}^{-}\left(I_{2}^{-} f\right)=$ $I_{3}(f)$.

Observe that if $l \in R_{\text {prop }}\left(B_{1}, I_{1}\right)$ such that $l(x)=I_{2}^{-}\left(f_{x}\right)$ whenever $f_{x} \in$ $R_{\text {prop }}\left(B_{2}, I_{2}\right)$, then $I_{1}(l)=I_{3}(f)$ and $I_{1}^{-}(|g-l|)=0$.

Remarks 1. 1. In general $I_{3}^{+}(f) \geq I_{1}^{+}\left(I_{2}^{+} f\right)$ is false for all $f \in \overline{\mathbb{R}}^{X_{3}}$, by 3.4 and Example 2 below. Therefore, an analogue to Theorem 1 for summable functions of [2] is in general not true.

Nevertheless, if $I$ is monotone-net-continuous = Bourbaki's continuity condition, then Daniell $L^{1}(B, I) \subset$ Bourbaki extension $L^{\tau}$ and $I^{+}=$upper Bourbaki extension $I^{\tau}$. In this case, $I_{3}^{\tau} \geq I_{1}^{\tau}\left(I_{2}^{\tau}\right)$ and there is an analogue to Theorem 1 (see [2], [6] and [13], p. 186).

2. For arbitrary $I / B$ it is easy to show that if $\left(h_{n}\right) \subset B, 0 \leq h_{n+1} \leq h_{n}$, $n \in \mathbb{N}, I\left(h_{n}\right) \rightarrow 0$, as $n \rightarrow \infty$, then there exist $A_{k} \subset X$ such that $I^{-}\left(\chi_{A_{k}}\right)=0$, and that if $x \notin \bigcup_{1}^{\infty} A_{k}$, then $h_{n}(x) \rightarrow 0$, as $n \rightarrow \infty$.

In general, $\bigcup_{1}^{\infty} A_{k}$ is not an $I^{-}$-null set by 3.4 and Example 3 below. If $I$ is $\sigma$-continuous, then $\bigcup_{1}^{\infty} A_{k}$ is an $I^{\sigma}$-null set (see [9], p. 265).

2.2. Abstract Riemann integration. (5) For any $f \in \overline{\mathbb{R}}^{X_{j}}, j=1,2,3$, the corresponding localized functionals in the sense of Schäfke [14] are defined by

$$
I_{j, l}^{-}(f):=\sup \left\{I_{j}^{-}(f \wedge h) ; 0 \leq h \in B_{j}\right\},
$$

and for $f \in \overline{\mathbb{R}}^{X_{3}}, I_{2, l}^{-} f: X_{1} \rightarrow \overline{\mathbb{R}}$ is defined by $\left(I_{2, l}^{-} f\right)(x):=I_{2, l}^{-}\left(f_{x}\right)$ for each $x \in X_{1} . I_{j, l}^{-}$is monotone and subadditive on $\overline{\mathbb{R}}^{X_{j}}$.

In view of the definitions involved, we have

(6) $\left(I_{j, l}^{-}\right)_{j, l}=I_{j, l}^{-} \leq I_{j}$, and $I_{j, l}^{-}(f)=I_{j}(f)$ if $f \in \overline{\mathbb{R}}^{X_{j}}$ and $f \leq$ some $h \in B_{j}$.

(7) For $j=1,2,3$, the set $R_{1}\left(B_{j}, I_{j}\right)$ of $I_{j}$-integrable functions is defined as the closure of $B_{j}$ in $\overline{\mathbb{R}}^{X_{j}}$ with respect to the integral seminorm $I_{j, l}^{-}(||)$.

$R_{1}\left(B_{j}, I_{j}\right)$ is closed with respect to,$\pm \alpha \cdot(\alpha \in \mathbb{R}) ;||, \wedge, \vee$. $I_{j, l}^{-} / R_{1}\left(B_{j}, I_{j}\right)$ is a positive linear functional (= unique $I_{j, l}^{-}$-continuous extension of $\left.I_{j} / B_{j}\right)$.

By [3], $R_{1}\left(B_{j}, I_{j}\right)$ is the set of all $f \in \overline{\mathbb{R}}^{X_{j}}$ to which there exists a sequence $\left(h_{n}\right) \subset B_{j}$, which is a Cauchy sequence with respect to $I_{j}(|\cdot|)$ and with $h_{n} \rightarrow f\left(I_{j}^{-}\right)$; then $I_{j}(f):=\lim I\left(h_{n}\right), n \rightarrow \infty$. In general, $R_{\text {prop }} \subset R_{1}$ with 
coinciding integrals, and $\subset$ generally strict. For further properties of $R_{1}$ see [3] and [10].

In all that follows we assume the following condition

To $h \in B_{1}, g \in B_{2}$ there exists $l \in B_{3}$ such that $g(y) \leq l(x, y)$ if $h(x)>0$.

Lemma 2. Let $f \in \overline{\mathbb{R}}^{X_{3}}$ such that the following condition holds:

$$
\left|f_{x}\right| \leq g \in B_{2} \quad \text { for each } x \in X_{1} \text {. }
$$

Then, $I_{1, l}^{-}\left(I_{2, l}^{-} f\right) \leq I_{3, l}^{-}(f)$.

Proof. For $f_{x} \in \overline{\mathbb{R}}_{+}^{X_{2}}, x \in X_{1}$, we have with $(* *)$ and $(5)\left(I_{2, l}^{-} f\right)(x)=$ $\left(I_{2}^{-} f\right)(x)$, so that $I_{1, l}^{-}\left(I_{2, l}^{-} f\right) \leq I_{1, l}^{-}\left(I_{2}^{-} f\right):=\sup \left\{I_{1}^{-}\left(\left(I_{2}^{-} f\right) \wedge h\right) ; 0 \leq h \in B_{1}\right\}$.

Now, with $(*),\left(I_{2}^{-} f\right) \wedge h \leq I_{2}^{-} f \leq I_{2}^{-} f \wedge l$, where $l \in B_{3}$ and $f_{x} \leq$ $g \leq l_{x}$ for each $x \in X_{1}, h(x)>0$. Hence, with Lemma 1 and (5), we have $I_{1}^{-}\left(\left(I_{2}^{-} f\right) \wedge h\right) \leq I_{1}^{-}\left(I_{2}^{-} f \wedge l\right) \leq I_{3}^{-}(f \wedge l) \leq I_{3,1}^{-}(f)$, for all $0 \leq h \in B_{1}$, and we conclude the result.

Without (**) Lemma 2 becomes false by p. 270 of [5]. Here there exists $f \in \overline{\mathbb{R}}_{+}^{X_{3}}$ such that $I_{1, l}^{-}\left(I_{2, l}^{-} f\right)=\infty$ and $I_{3, l}^{-}(f)=0$.

Theorem 2 is obtained now in a similar way as Satz p. 141 of Hoffman [11] (see also Elsner [5]).

Theorem 2. Let $\left(X_{3}, B_{3}\right)$ be a product system, and let $f \in R_{1}\left(B_{3}, I_{3}\right)$ satisfying $(* *)$. Then the following assertions hold:

(i) There exist $A_{k} \subset X_{1}, k \in \mathbb{N}, I_{1,-}^{-}$-null sets, such that $f_{x} \in R_{1}\left(B_{2}, I_{2}\right)$ for each $x \in X_{1}-\bigcup_{1}^{\infty} A_{k}$.

(ii) There exists $g \in R_{1}\left(B_{1}, I_{1}\right)$ defined by $I_{2, l}^{-}\left(f_{x}\right)$ if $f_{x} \in R_{1}\left(B_{2}, I_{2}\right)$, and such that $I_{1, l}^{-}(g)=I_{3, l}^{-}(f)$, i.e. $I_{3, l}^{-}(f)=I_{1, l}^{-}\left(I_{2, l}^{-} f\right)$.

Proof. (i) By (7), for $f \in R_{1}\left(B_{3}, I_{3}\right)$, given $\varepsilon>0$ there exists $g \in B_{3}$ such that $I_{3, l}^{-}(|f-g|)<\varepsilon$.

For each $x \in X_{1}$ set $\varphi(x):=\inf \left\{I_{2, l}^{-}\left(\left|f_{x}-h\right|\right)\right.$, for all $\left.h \in B_{2}\right\}$ and set $A_{k}:=\left\{x \in X_{1} ; \varphi(x) \geq \frac{1}{k}\right\}, k \in \mathbb{N}$. By virtue of Lemma 2 , it can be easily proved that the sets $A_{k}, k \in \mathbb{N}$, are $I_{1, l}^{-}$-null, and (i) follows immediately.

To prove (ii) it suffices to see that there is $\left(I_{2, l}^{-} g_{n}\right) \subset B_{1}$ such that $I_{2, l}^{-} g_{n} \rightarrow$ $g\left(I_{1, l}^{-}\right)$, where $\left(g_{n}\right) \subset B_{3}$ and $I_{3, l}^{-}\left(\left|g_{n}-f\right|\right) \rightarrow 0$, as $n \rightarrow \infty$.

In fact, a calculation analogous to the proof given in [11] (Hauptsatz, p. 139, with "Fubini-integral norms"), and having in mind the properties stated in (6) and (7), permits to show the inequality

$$
I_{1, l}^{-}\left(\left|\left(I_{2, l}^{-} g_{n}\right)-g\right|\right) \leq 3 I_{1, l}^{-}\left(I_{2, l}^{-}\left|g_{n}-f\right|\right) \leq 3 I_{3, l}^{-}\left(\left|g_{n}-f\right|\right) \text {. }
$$

Besides, $I_{1, l}^{-}\left(I_{2, l}^{-} g_{n}\right) \rightarrow I_{1, l}^{-}(g)$, as $n \rightarrow \infty$, and $I_{1, l}^{-}(g)=I_{3, l}^{-}(f)$.

Remarks 2. 1. In the above statement usually all assumptions are essential. There exist counterexamples for the $\lambda \times \mu$-case (additive measure space, see 3.1. below) in [5], Bem.4.b, and 4.c.p.270. Similar examples show that one cannot substitute $\left|f_{x}\right| \leq g$ for $f_{x} \leq g$ in $(* *)$. 
2. There are simple examples of $f \in R_{1}(\lambda \times \mu, \mathbb{R})$ with $(* *)$, but $f \notin$ $R_{\text {prop }}(\lambda \times \mu, \mathbb{R})$ :

$$
f=\chi_{X_{1} \times M}, M \in \text { ring } \Omega_{2}, \text { with } \mu\left(X_{1}\right)=\infty .
$$

3. Let us finally remark that our results can be reformulated for Banach spacevalued functions, using $f \cap g:=\|f\|^{-1}(\|f\| \wedge g) f$, with $f: X \rightarrow E=$ Banach space, $g \in \mathbb{R}_{+}^{X}$, of [9], p. 327 .

\section{Applications AND EXAMPles}

1. If $\Omega$ is a semiring of sets $\subset X$ and $\mu: \Omega \rightarrow[0, \infty[$ is additive, then $B=B_{\Omega}:=$ real-valued step functions over $\Omega$ and $I=I_{\mu}:=\int \cdot d_{\mu}$ satisfying (1).

Then the proper Riemann- $\mu$-integrable functions $R_{\text {prop }}(\mu, \mathbb{R})=I_{\mu}^{-}$-closure of $B_{\Omega}$ in $\mathbb{R}^{X}$, in the sense of Aumann [1], p. 448.

The space of abstract Riemann- $\mu$-integrable functions $R_{1}(\mu, \mathbb{R})$ was presented essentially by Loomis [12]. For Banach space-valued functions it has been introduced by Dunford-Schwartz [4], and in more general form by Günzler [8], [9]. $R_{\text {prop }}(\mu, \mathbb{R}) \subset$ Dunford-Schwartz integral $L(X, \Omega, \mu, \mathbb{R}) \subset R_{1}(\mu, \overline{\mathbb{R}})$, with coinciding integrals; all $\subset$ are in general strict (see Lemma 9 of [10] and [9], pp. 199, 70).

In Gould [7], Stone's axiom $B \wedge 1 \subset B$ is assumed, so by [8] his results are already subsumed by the abstract Riemann integral (see, for example, [9], pp. $57,268)$.

If $\Omega_{1}$ and $\Omega_{2}$ are semirings of sets from $X_{1}$ and $X_{2}$, and $\mu_{1}$ and $\mu_{2}$ are additive measures on $\Omega_{1}$ and $\Omega_{2}$, respectively, one can construct a product additive measure $\mu_{3}$ in the set $X_{3}:=X_{1} \times X_{2}$ and the induced integral $I_{\mu_{3}}$.

If we set $\Omega_{3}:=\left\{A_{1} \times A_{2} ; A_{j} \in \Omega_{j}, j=1,2\right\}$, then, $\mu_{3}\left(A_{1} \times A_{2}\right):=\mu_{1}\left(A_{1}\right) \cdot$ $\mu_{2}\left(A_{2}\right)=I_{\mu_{3}}\left(\chi_{A_{1} \times A_{2}}\right)$. See [13], $\S 16 ;[11]$, p. 125 .

2. If $B=B_{\Omega}$ with $\Omega=\sigma$-ring and $I=I_{\mu}$ with $\mu \quad \sigma$-additive, then $R_{1}(\mu, \overline{\mathbb{R}})=L^{1}(\mu, \overline{\mathbb{R}}) \quad(:=$ Lebesgue- $\mu$-integrable functions $)$, and $f_{n} \rightarrow f \mu$ almost everywhere implies $f_{n} \rightarrow f\left(I_{\mu}^{-}\right)$for $\mu$-measurable $f_{n}$, by [9], p. 265; and we get the usual Lebesgue convergence theorems.

In [5] Elsner has given a very thorough and interesting treatment of the Fubini theorem for the abstract Riemann- $\mu$-integral. Our results contain properly that of [5], for which we obtain simplified proofs. Indeed, Example 1 below shows that there exist functions for which Theorem 1 is applicable, but not the corresponding result of [5] or even [11], p. 129.

Observe that for the $\lambda \times \mu$-case, $(*)$ holds and $(* *)$ means that $|f|$ is bounded and there exists $P \in$ ring generated $\Omega_{2}$ such that $\operatorname{supp}(f) \subset X_{1} \times P$.

In [14] integration with local Loomis-Schäfke integral seminorms is obtained. With (7), we have $R_{1}(B, I) \cap \mathbb{R}^{X}=$ Schäfke local $I_{1}^{-}$-closure of $B$, and $R_{1}(B, I) \cap \mathbb{R}^{x}=$ "one-sided completion" of Loomis [12], p. 170.

3. We denote by $B_{1} \otimes B_{2}$ the vector space of functions on $X_{3}$ generated by the family $\left\{g \otimes h ; g \in B_{1}, h \in B_{2}\right\}$, where $(f \otimes k)(x, y):=f(x) \cdot k(y)$ for arbitrary $f$ and $k$ on $X_{1}$ and $X_{2}$, respectively. If $|f| \in B_{1} \otimes B_{2}$ whenever $f \in B_{1} \otimes B_{2}$, then $B_{1} \otimes B_{2}$ is a product system with respect to $\left(B_{1}, I_{1}\right)$ and $\left(B_{2}, I_{2}\right)$ (see [13], $\S 15 ;[6]$, p. 187), and Sections 1 and 2 are applicable.

4. Using Examples 1-3 below it is not difficult to check that there are finitely additive $\lambda, \mu$ on rings and $f, g, h_{n}$ with $0 \leq f=I_{\mu \times \mu}^{-}$-null functions, but 
$f_{x} \in R_{\text {prop }}(\mu, \mathbb{R})$ for no $x \in X_{1}$ (Example 1$) ; g \in R_{1}(\lambda \times \mu, \mathbb{R})$, all $g^{y} \in B_{1}$, $I_{1}\left(g^{y}\right) \in B_{2}$, but $\int g d(\lambda \times \mu) \neq \int\left(\int g d \lambda\right) d \mu$ (Example 2$) ; 0 \leq h_{n+1} \leq h_{n}, \int h_{n}$ $d \mu \rightarrow 0$, but $h_{n}(x) \rightarrow 0$ for no $x$ (Example 3). $g$ can be found in Elsner [5], p. 270.

Example 1. Let $X_{1}=X_{2}=\mathbb{N}, \Omega_{1}=\Omega_{2}=\{\mathbb{N}-E, E$; finite set $\subset \mathbb{N}\}$, $\mu_{1}=\mu_{2}=\mu$ finitely additive measure, such that $\mu(E):=0, \mu(\mathbb{N}):=1$.

Let $X_{3}:=\mathbb{N} \times \mathbb{N}, \Omega:=\left\{X_{3}-E, E ; E\right.$ finite set $\left.\subset X_{3}\right\}$, and $\nu: \Omega \rightarrow \mathbb{R}$, $\nu(E):=0, \nu\left(X_{3}\right):=1$.

Example 2. Let $\left.\left.\left.\left.X_{1}=\mathbb{R}, \Omega_{1}:=\{] a, b\right] ; a, b \in \mathbb{R}, a \leq b\right\}, \lambda(] a, b\right]\right):=b-$ $a ; X_{2}=\mathbb{N}, \Omega_{2}:=\{\mathbb{N}-E, E ; E$ finite set $\subset \mathbb{N}\}, \mu(\mathbb{N})=1, \mu(E)=0$, and $X_{3}:=X_{1} \times X_{2}=\mathbb{R} \times \mathbb{N}$. Let $I_{1}=I_{\lambda}, I_{2}=I_{\mu}, B_{1}=B_{\Omega_{1}}, B_{2}=B_{\Omega_{2}}$, $B_{3}:=B_{\Omega_{1} \times \Omega_{2}}, I_{3}:=I_{1} \circ I_{2}=\int \cdot d(\lambda \times \mu)$.

Example 3. Let $X=\mathbb{N}, \Omega=\{\mathbb{N}-E, E ; E$ finite set $\subset \mathbb{N}\}, \mu(\mathbb{N})=1$, $\mu(E)=0, B=B_{\Omega}$, and $I=I_{\mu}$.

\section{ACKNOWLEDGMENT}

We thank Professor H. Günzler for comments on and improvements in the obtain results.

\section{REFERENCES}

1. G. Aumann, Integralerweiterungen mittels Normen, Arch. Math. (Basel) 3 (1952), 441-450.

2. P. Bobillo Guerrero and M. Díaz Carrillo, Summable and integrable functions with respect to any Loomis system, Arch. Math. (Basel) 49 (1987), 245-256.

3. M. Díaz Carrillo and P. Muñoz Rivas, Finitely additive integration: integral extension with local-convergence, Ann. Sci. Math. Québec 17 (1993), 1-9.

4. N. Dunford and J. T. Schwartz, Linear operators. I, Interscience, New York, 1957.

5. J. Elsner, Zum "Satz von Fubini" für ein abstraktes Riemann-integral, Math. Z. 141 (1975), 265-278.

6. K. Floret, Maß-und Integrationstheorie, Teubner, Stuttgart, 1981.

7. G. G. Gould, The Daniell-Bourbaki integral for finitely additive measures, Proc. London Math. Soc. 16 (1966), 297-320.

8. H. Günzler, Linear functionals which are integrals, Rend. Sem. Mat. Fis. Milano XVIII (1977), 167-176.

9. __ Integration, Bibliogr. Institut, Mannheim, 1985. 10. $\underset{77-94}{ }$. Convergence theorems for a Daniell-Loomis integral, Math. Pannonica 2/2 (1991),

11. D. Hoffmann, Zum “Satz von Fubini”, J. Reine Angew. Math. 298 (1977), 138-145.

12. L. H. Loomis, Linear functionals and content, Amer. J. Math. 76 (1954), 168-182.

13. W. F. Pfeffer, Integrals and measures, Dekker, New York, 1977.

14. F. W. Schäfke, Lokale Integralnormen and verallgemeinerte uneigentlich Riemann-StiltjesIntegrals, J. Reine Angew. Math. 289 (1977), 118-134.

Departamento de Análisis Matemático, Universidad de Granada, 18071 Granada, 